Journal of

NONLINEAR

MATHEMATICAL

PHYSICS

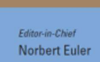

Norbert Euler

(

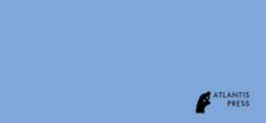

\begin{abstract}
Journal of Nonlinear Mathematical Physics
\end{abstract}

ISSN (Online): 1776-0852 ISSN (Print): 1402-9251

Journal Home Page: https://www.atlantis-press.com/journals/inmp

\title{
On integrability of the Szekeres system. I
}

Anna Gierzkiewicz, Zdzisław A. Golda

To cite this article: Anna Gierzkiewicz, Zdzisław A. Golda (2016) On integrability of the Szekeres system. I, Journal of Nonlinear Mathematical Physics 23:4, 494-506, DOI: https://doi.org/10.1080/14029251.2016.1237199

To link to this article: https://doi.org/10.1080/14029251.2016.1237199

Published online: 04 January 2021 


\title{
On integrability of the Szekeres system. I
}

\author{
Anna Gierzkiewicz \\ Department of Applied Mathematics, University of Agriculture in Kraków, \\ ul. Balicka 253c, 30-198 Kraków, Poland \\ Astronomical Observatory of the Jagiellonian University \\ ul. Orla 171, 30-244 Kraków, Poland \\ anna.gierzkiewicz@ur.krakow.pl \\ Zdzisław A. Golda \\ Astronomical Observatory of the Jagiellonian University \\ ul. Orla 171, 30-244 Kraków, Poland \\ zdzislaw.golda@uj.edu.pl
}

Received 24 March 2016

Accepted 25 July 2016

\begin{abstract}
The Szekeres system is a four-dimensional system of first-order ordinary differential equations with nonlinear but polynomial (quadratic) right-hand side. It can be derived as a special case of the Einstein equations, related to inhomogeneous and nonsymmetrical evolving spacetime. The paper shows how to solve it and find its three global independent first integrals via Darboux polynomials and Jacobi's last multiplier method. Thus the Szekeres system is completely integrable. Its two-dimensional subsystem is also investigated: we present its solutions explicitly and discuss its behaviour at infinity.
\end{abstract}

Keywords: First integral; Szekeres system; Darboux polynomial; Jacobi Last Multiplier; Poincaré compactification

2000 Mathematics Subject Classification: 37J35, 83C15, 83C20

\section{Introduction. The Szekeres system}

Szekeres in [11] presented a new class of exact solutions of Einstein equations for irrotational dust. The limiting case of Szekeres models are Lemaître-Tolman models, which in turn involve Friedmann-Lemaître-Robertson-Walker models as their limiting case [2].

The Szekeres models can be written down in the form of real autonomous fourth-order dynamical system:

$$
\left\{\begin{array}{l}
\rho^{\prime}=-\theta \rho, \\
\theta^{\prime}=-\frac{1}{3} \theta^{2}-6 \sigma^{2}-\frac{1}{2} \rho, \\
\sigma^{\prime}=\sigma^{2}-\frac{2}{3} \theta \sigma-E, \\
E^{\prime}=-3 E \sigma-\theta E-\frac{1}{2} \rho \sigma,
\end{array}\right.
$$

where the variables dependent on the time $t$ with respect to which they are differentiated are respectively: energy density $\rho$, expansion scalar $\theta$, shear $\sigma$, and the electrical component of the Weyl tensor $E$. 
When we put two of the four variables as equal to zero, $\sigma=0$ and $E=0$, then the Szekeres system (1.1) reduces to the following dynamical system

$$
\left\{\begin{array}{l}
\rho^{\prime}=-\theta \rho, \\
\theta^{\prime}=-\frac{1}{3} \theta^{2}-\frac{1}{2} \rho,
\end{array}\right.
$$

which describes the Friedmann-Lemaître-Robertson-Walker models.

It is worth noting that the Szerekes models are a particular case of more general Silent Universe models [3], which are described by a sixth order dynamical system with energy density $\rho$, expansion scalar $\theta$, two independent eigenvalues $\sigma_{1}$ and $\sigma_{2}$ of the traceless shear tensor $\sigma_{a b}$, and eigenvalues $E_{1}$ and $E_{2}$ of the traceless component of the Weyl tensor $E_{a b}$ as variables. If $\sigma_{1}=\sigma_{2}=\sigma$ and $E_{1}=E_{2}=E$, then the Silent Universe models reduce to the Szekeres system (1.1).

Recently there can be noted ever growing interest in Szekeres's solutions. They have been applied in cosmology in describing formation and evolution of structure in the Universe, as well as in investigating effects related to propagation of light in nonhomogeneous universe models (see e.g. $[1,10])$.

Our purpose is to show the complete integrability of the system (1.1) by finding its three independent first integrals. The paper consists of seven sections and an appendix. In the second section, we introduce the notion of first integral. Section 3 contains the description of the Darboux polynomials method and two first integrals of the system (1.1) resulting from the method's application. Next, in Section 4, one can find the key transformation of the system (1.1), the Jacobi's last multiplier method, and the third independent first integral.

Sections 5 and 6 describe the way to solve the transformed system (4.1) directly and present some of its special solutions, contained in an invariant two-dimensional subspace. In Section 7, we provide the conclusions and pose a number of further questions. Appendix A explains the Poincaré compactification of the invariant two-dimensional subsystem of (4.1), presented on Fig. 1.

\section{Preliminaries}

We introduce our notions with respect to a system of $n$ autonomous ordinary differential equations defined on an open set $U \subset \mathbb{R}^{n}$

$$
\dot{\mathbf{x}}=\mathbf{f}(\mathbf{x}),
$$

where $\mathbf{x}=\left(x_{1}, \ldots, x_{n}\right), \mathbf{f}=\left(f_{1}, \ldots, f_{n}\right)$, and $f_{i}$ is a $\mathscr{C}^{1}$ real-valued function on $U$ for every $i=1, \ldots, n$.

By a (global) first integral of a system (2.1) we understand a $\mathscr{C}^{1}$ function $I=I(\mathbf{x}): V \rightarrow \mathbb{R}$, defined on an open dense subset $V \subset U$, such that

$$
\delta_{\mathbf{f}}(I):=\sum_{i=1}^{n} f_{i} \frac{\partial I}{\partial x_{i}}=0 .
$$

Let $I_{1}, \ldots, I_{k}$ be $k$ first integrals of (2.1). We call them independent first integrals if there exists an open dense subset $V \subset U$ such that $V \subset \operatorname{dom} I_{i}$ for every $i=1, \ldots, k$ and the rank of the matrix

$$
\left[\begin{array}{ccc}
\frac{\partial I_{1}}{\partial x_{1}}(a) & \ldots & \frac{\partial I_{1}}{\partial x_{n}}(a) \\
\vdots & \ddots & \vdots \\
\frac{\partial I_{k}}{\partial x_{1}}(a) & \ldots & \frac{\partial I_{k}}{\partial x_{n}}(a)
\end{array}\right]
$$


is equal to $k$ for every $a \in V$. The definition of a first integral $I$ restricts the dimension of the space of possible gradient vectors $\left(\frac{\partial I}{\partial x_{1}}(a), \ldots, \frac{\partial I}{\partial x_{n}}(a)\right)$ at a point $a$ to $n-1$. Therefore, the system (2.1) has at most $n-1$ independent global first integrals.

\section{Darboux polynomials method}

Two rational first integrals for (1.1) can be found by the well-known method of Darboux [4], in a broadly developed form (see e.g. [6]). The point is to find as many as possible Darboux polynomials for the system.

Definition 3.1. A Darboux polynomial of the system (2.1) is a polynomial $J=J(\mathbf{x}) \in \mathbb{R}[\mathbf{x}]$ fulfilling an 'eigen-equation'

$$
\delta_{\mathbf{f}}(J)=\mu J
$$

with some polynomial $\mu=\mu(\mathbf{x}) \in \mathbb{R}[\mathbf{x}]$, called a cofactor.

Note that a Darboux polynomial $J$ involves some geometrical information on the system (2.1), as its zero-level sets are invariant hypersurfaces for the flow defined by the vector field $\delta_{\mathrm{f}}$. It follows straight from the fact that

$$
J(\mathbf{x})=0 \quad \Longrightarrow \quad \delta_{\mathbf{f}}(J)(\mathbf{x})=\mu(\mathbf{x}) J(\mathbf{x})=0 .
$$

However, most important for us is the fact that sufficiently many Darboux polynomials form a first integral.

Theorem 3.1 (Darboux [5]). Let $\mathbf{f} \in \mathbb{C}[\mathbf{x}]^{n}$ be a polynomial vector field of degree $d$ and assume that $\delta_{\mathrm{f}}$ admits $q$ Darboux polynomials $J_{1}, \ldots, J_{q}$. Then, if $q>\left(\begin{array}{c}n+d-1 \\ n\end{array}\right)$, the system admits a first integral of the form

$$
I=J_{1}^{\lambda_{1}} \cdot \ldots \cdot J_{q}^{\lambda_{q}}, \quad \lambda_{i} \in \mathbb{C} .
$$

Applying this theorem to the real case of the Szekeres system, we need at least $\left(\begin{array}{c}4+2-1 \\ 4\end{array}\right)=5$ Darboux polynomials to be sure to obtain a first integral. Here, however, just four are sufficient. Algorithmic searching for a Darboux polynomial $J$ consists in fixing the degree of $J$ and solving a (overdetermined) system of linear equations for its indefinite coefficients. Direct computation in Mathematica [8] results in two Darboux polynomials of degree one for the Szekeres system:

$$
\begin{array}{ll}
J_{11}=\rho, & \mu_{11}=-\theta, \\
J_{12}=6 E+\rho, & \mu_{12}=-\theta-3 \sigma,
\end{array}
$$

and two of degree two:

$$
\begin{array}{ll}
J_{21}=-3(6 E+\rho)+(\theta+3 \sigma)^{2}, & \mu_{21}=-\frac{2}{3} \theta-2 \sigma \\
J_{22}=9 E+\theta^{2}-3 \rho-3 \theta \sigma-18 \sigma^{2}, & \mu_{22}=-\frac{2}{3} \theta+\sigma .
\end{array}
$$

There are no independent Darboux polynomials of degree three. 
Following the Darboux theory, we check whether there exists a first integral of the form

$$
J_{11}^{\lambda_{11}} J_{12}^{\lambda_{12}} J_{21}^{\lambda_{21}} J_{22}^{\lambda_{22}}, \quad \lambda_{i j} \in \mathbb{R} .
$$

It turns out that for $\lambda_{12}=-\frac{1}{3}\left(\lambda_{11}+2 \lambda_{21}\right), \lambda_{22}=-\lambda_{11}$ one obtains first integrals. Such $\lambda \mathrm{s}$ span a two-dimensional vector space, so we get two families of independent first integrals. Let us fix one of each family:

$$
\begin{array}{ll}
I_{1}=\frac{-3(6 E+\rho)+(\theta+3 \sigma)^{2}}{(6 E+\rho)^{2 / 3}} & \text { for } \lambda_{11}=0, \lambda_{21}=1, \\
I_{2}=\frac{(6 E+\rho)^{1 / 3}\left(9 E+\theta^{2}-3 \rho-3 \theta \sigma-18 \sigma^{2}\right)}{\rho} & \text { for } \lambda_{11}=-1, \lambda_{21}=0 .
\end{array}
$$

One can easily check that the integrals are independent on the common domain $\left\{J_{11} \cdot J_{12} \cdot J_{21} \cdot J_{22} \neq\right.$ $0\}$, which is an open dense subset of $\mathbb{R}^{4}$, invariant under the flow. Note that $\lambda \mathrm{s}$ can be also chosen so that to get rational integrals (e.g. $I_{1}^{3}$ and $I_{2}^{3}$ ).

\section{Jacobi multiplier}

In order to prove the complete integrability of the four-dimensional system (1.1), we need an additional global first integral, functionally independent of $I_{1}, I_{2}$. We apply here the Jacobi's last multiplier method.

\subsection{Change of variables}

First we introduce new variables

$$
\varepsilon:=6 E+\rho, \quad \beta:=-3 \sigma-\theta .
$$

The new variables are related to one of the Darboux polynomials of degree one: $\varepsilon=J_{12}, \beta=\mu_{12}$. The system (1.1) in the variables $(\rho, \theta, \beta, \varepsilon)$ can be rewritten as follows:

$$
\left\{\begin{array}{l}
\rho^{\prime}=-\theta \rho, \\
\theta^{\prime}=-\theta^{2}-\frac{2}{3} \beta^{2}-\frac{4}{3} \beta \theta-\frac{1}{2} \rho, \\
\beta^{\prime}=\frac{1}{3} \beta^{2}+\frac{1}{2} \varepsilon, \\
\varepsilon^{\prime}=\varepsilon \beta .
\end{array}\right.
$$

We also transform the Darboux polynomials:

$$
\begin{array}{llrl}
j_{11} & =\rho, & \mu_{11} & =-\theta, \\
j_{12} & =\varepsilon, & \mu_{12} & =\beta, \\
j_{21} & =-\frac{1}{3} \beta^{2}+\varepsilon, & \mu_{21} & =\frac{2}{3} \beta, \\
j_{22} & =\frac{1}{6}\left(4 \beta^{2}+6 \beta \theta-3 \varepsilon+9 \rho\right), & \mu_{22} & =-\frac{1}{3} \beta-\theta,
\end{array}
$$

and the previously chosen two independent first integrals now get the form $I_{1,2}(\rho, \theta, \sigma, E) \mapsto$ $i_{1,2}(\rho, \theta, \beta, \varepsilon)$, where:

$$
i_{1}:=\frac{3 \varepsilon-\beta^{2}}{\varepsilon^{2 / 3}}, \quad i_{2}:=\frac{\sqrt[3]{\varepsilon}\left(4 \beta^{2}+6 \beta \theta-3 \varepsilon+9 \rho\right)}{6 \rho} .
$$




\subsection{Outline of the method}

The Jacobi's last multiplier method for an $n$-dimensional system $\dot{\mathbf{x}}=\mathbf{f}(\mathbf{x})$ allows to find an additional first integral if $n-2$ integrals are known. In the original formulation, the system is locally volume-preserving, but the theorem giving this integral explicitly is also valid if the system admits a Jacobi multiplier $M$.

Definition 4.1. A Jacobi multiplier for the system (2.1) is a non-zero $\mathscr{C}^{1}$ real function $M=M(\mathbf{x})$ so that

$$
\operatorname{div}(M \mathbf{f})=\sum_{i=1}^{n} \frac{\partial\left(M f_{i}\right)}{\partial x_{i}}=0
$$

In other words, the system multiplied by $M$ becomes a volume-preserving one.

There are several methods for finding such a multiplier. If the system is polynomial, one often searches for a multiplier of the form $\mathbf{x}^{\alpha}=x_{1}^{\alpha_{1}} \cdot \ldots \cdot x_{n}^{\alpha_{n}}$ by solving a system of linear equations for $\alpha$ s. The method is presented briefly in $[5]^{\mathrm{a}}$.

In the first place, we present a system $S$ of $n$ first-order ordinary differential equations in the matrix form

$$
S: \quad \dot{x}_{i}=x_{i} \sum_{j=1}^{m} A_{i j} \prod_{k=1}^{n} x_{k}^{B_{j k}}, \quad i=1, \ldots, n
$$

where $A \in M_{n, m}(\mathbb{R}), B \in M_{m, n}(\mathbb{K})$. Typically, $\mathbb{K}=\mathbb{N}, \mathbb{Z}$ or $\mathbb{Q}$. For a system with a polynomial vector field, we have in general $\mathbb{K}=\mathbb{N} \cup\{-1\}$. The matrices $A$ and $B$ are determined uniquely if we do not allow zero line vectors and order the monomials lexicographically. We also specify $S=S(A, B, \mathbf{x}, t)$.

The Szekeres system (4.1) in the new variables is expressed by $S=S(A, B,(\rho, \theta, \beta, \varepsilon), t)$, where

$$
A=\left(\begin{array}{ccccc}
0 & 0 & 0 & -1 & 0 \\
-\frac{2}{3} & 0 & -\frac{4}{3} & -1 & -\frac{1}{2} \\
0 & \frac{1}{2} & \frac{1}{3} & 0 & 0 \\
0 & 0 & 1 & 0 & 0
\end{array}\right), \quad B=\left(\begin{array}{cccc}
0 & -1 & 2 & 0 \\
0 & 0 & -1 & 1 \\
0 & 0 & 1 & 0 \\
0 & 1 & 0 & 0 \\
1 & -1 & 0 & 0
\end{array}\right)
$$

Theorem 4.1 (Goriely [5], Prop. 4.11). Let $S(A, B, \mathbf{x}, t)$ be a polynomial ${ }^{\mathrm{b}}$ system and $M=B A$. Define $\mathbf{v} \in \mathbb{C}^{m}$ to be the vector of components $v_{i}=-M_{i i}-\sum_{j=1}^{n} A_{j i}$.

If $\boldsymbol{\eta} \cdot \mathbf{v}=0, \forall \boldsymbol{\eta} \in \operatorname{Ker}(M)$, then the system $S(A, B)$ admits a Jacobi multiplier of the form $\mathbf{x}^{\boldsymbol{\alpha}}$, where $\boldsymbol{\alpha}$ is a solution of $A^{T} \boldsymbol{\alpha}=\mathbf{v}$.

Using this method, we obtain $\boldsymbol{\alpha}=\left(-3,0,0,-\frac{1}{3}\right)$, which corresponds to the multiplier for (4.1):

$$
M(\rho, \theta, \beta, \varepsilon)=\rho^{-3} \varepsilon^{-1 / 3} .
$$

With $M, i_{1}, i_{2}$, the assumptions of the following theorem are fulfilled:

Theorem 4.2 (Jacobi [5]). Consider an n-dimensional vector field $\dot{\mathbf{x}}=\mathbf{f}(\mathbf{x})$ and assume that it admits a Jacobi multiplier $M=M(\mathbf{x})$ and $(n-2)$ first integrals $I_{i}(\mathbf{x})=c_{i}, i=1, \ldots, n-2$. Then the

aProp. 4.11 in [5] actually involves an error. We have corrected it here.

${ }^{\mathrm{b}}$ Originally, quasinomial, which is too general for this case. 
system admits an extra first integral given by

$$
I_{n-1}=\int \frac{\tilde{M}}{\tilde{\Delta}}\left(\tilde{f}_{n} \mathrm{~d} x_{n-1}-\tilde{f}_{n-1} \mathrm{~d} x_{n}\right)
$$

where $(\sim)$ denotes quantities expressed in the variables $\left(c_{1}, \ldots, c_{n-2}, x_{n-1}, x_{n}\right)$ and

$$
\Delta=\left|\begin{array}{cccc}
\partial_{x_{1}} I_{1} & \partial_{x_{2}} I_{1} & \ldots & \partial_{x_{n-2}} I_{1} \\
\partial_{x_{1}} I_{2} & \partial_{x_{2}} I_{2} & \ldots & \partial_{x_{n-2}} I_{2} \\
\vdots & \vdots & \ddots & \vdots \\
\partial_{x_{1}} I_{n-2} & \partial_{x_{2}} I_{n-2} & \ldots & \partial_{x_{n-2}} I_{n-2}
\end{array}\right|
$$

\subsection{Third integral of the system (1.1)}

Direct computation leads to the third integral of the system (4.1):

$$
i_{3}=-\frac{\sqrt{i_{1}^{3}} \sqrt[3]{\varepsilon}(-\beta-2 \theta)}{\rho}+\frac{3 \beta \sqrt{i_{1}} i_{2}}{\varepsilon^{2 / 3}}-3\left(3 i_{2}-i_{1}\right) \arctan \left(\frac{-\beta}{\sqrt{i_{1}} \sqrt[3]{\varepsilon}}\right)
$$

Substituting $i_{1} \mapsto\left(3 \varepsilon-\beta^{2}\right) \varepsilon^{-2 / 3}$ and then $\varepsilon \mapsto 6 E+\rho, \beta \mapsto-3 \sigma-\theta$, we finally obtain the first integral for the original system (1.1):

$$
\begin{array}{r}
I_{3}=\frac{3\left(-E\left(18 E+2 \theta^{2}+3 \rho\right)+3 \theta \sigma(2 E+\rho)+9 \sigma^{2}(4 E+\rho)\right) \arctan \left(\frac{\theta+3 \sigma}{\sqrt{18 E-(\theta+3 \sigma)^{2}+3 \rho}}\right)}{\rho(6 E+\rho)^{2 / 3}}+ \\
+\frac{\sqrt{18 E-(\theta+3 \sigma)^{2}+3 \rho}\left(9 E(\sigma-\theta)+\sigma\left((\theta+3 \sigma)^{2}+6 \rho\right)\right)}{\rho(6 E+\rho)^{2 / 3}}
\end{array}
$$

The Jacobi theorem, however, gives the third integral just locally. One can readily notice that $I_{3}$ is defined for $\rho(6 E+\rho)=J_{11} \cdot J_{12} \neq 0-$ which is not a problem here, and $\left(18 E-(\theta+3 \sigma)^{2}+3 \rho\right)=$ $-J_{21}>0$, which restricts the domain essentially.

Let us extend it to the common domain $\left\{J_{11} \cdot J_{12} \cdot J_{21} \cdot J_{22} \neq 0\right\}$. We use the fact that the above formula is a complex-valued function and that $\arctan (i x)=-i \operatorname{artanh}(x)$. The third integral $I_{3}$ can be thus extended as follows:

- for $J_{11} \cdot J_{12} \cdot J_{22} \neq 0, \quad J_{21}<0$ :

$$
\begin{gathered}
\frac{3\left(-E\left(18 E+2 \theta^{2}+3 \rho\right)+3 \theta \sigma(2 E+\rho)+9 \sigma^{2}(4 E+\rho)\right) \arctan \left(\frac{\theta+3 \sigma}{\sqrt{18 E-(\theta+3 \sigma)^{2}+3 \rho}}\right)}{\rho(6 E+\rho)^{2 / 3}}+ \\
+\frac{\sqrt{18 E-(\theta+3 \sigma)^{2}+3 \rho}\left(9 E(\sigma-\theta)+\sigma\left((\theta+3 \sigma)^{2}+6 \rho\right)\right)}{\rho(6 E+\rho)^{2 / 3}}
\end{gathered}
$$


A. Gierzkiewicz and Z. A. Golda / On integrability of the Szekeres system. I

- for $J_{11} \cdot J_{22} \neq 0, \quad J_{21}>0, \quad J_{12}<0$ :

$$
\begin{gathered}
\frac{3\left(-E\left(18 E+2 \theta^{2}+3 \rho\right)+3 \theta \sigma(2 E+\rho)+9 \sigma^{2}(4 E+\rho)\right) \operatorname{artanh}\left(\frac{\theta+3 \sigma}{\sqrt{-\left(18 E-(\theta+3 \sigma)^{2}+3 \rho\right)}}\right)}{\rho(6 E+\rho)^{2 / 3}}- \\
-\frac{\sqrt{-\left(18 E-(\theta+3 \sigma)^{2}+3 \rho\right)}\left(9 E(\sigma-\theta)+\sigma\left((\theta+3 \sigma)^{2}+6 \rho\right)\right)}{\rho(6 E+\rho)^{2 / 3}},
\end{gathered}
$$

- for $J_{11} \cdot J_{22} \neq 0, \quad J_{21}>0, \quad J_{12}>0$ :

$$
\begin{gathered}
\frac{3\left(-E\left(18 E+2 \theta^{2}+3 \rho\right)+3 \theta \sigma(2 E+\rho)+9 \sigma^{2}(4 E+\rho)\right) \operatorname{arcoth}\left(\frac{\theta+3 \sigma}{\sqrt{-\left(18 E-(\theta+3 \sigma)^{2}+3 \rho\right)}}\right)}{\rho(6 E+\rho)^{2 / 3}}- \\
-\frac{\sqrt{-\left(18 E-(\theta+3 \sigma)^{2}+3 \rho\right)}\left(9 E(\sigma-\theta)+\sigma\left((\theta+3 \sigma)^{2}+6 \rho\right)\right)}{\rho(6 E+\rho)^{2 / 3}}
\end{gathered}
$$

Furthermore, we separate cases $J_{12}<0$ and $J_{12}>0$, because artanh as a real function is defined only on the interval $(-1,1)$ and the domain of arcoth is $(-\infty,-1) \cup(1,+\infty)$. One can check that

$$
\left|\frac{\theta+3 \sigma}{\sqrt{-\left(18 E-(\theta+3 \sigma)^{2}+3 \rho\right)}}\right|<1 \quad \Longleftrightarrow 6 E+\rho=J_{12}<0 .
$$

As mentioned before, the domain $\left\{J_{11} \cdot J_{12} \cdot J_{21} \cdot J_{22} \neq 0\right\}$, common for $I_{1}, I_{2}, I_{3}$, is an open, dense and invariant set and therefore we obtain three global independent first integrals for the Szekeres system. Hence we can state the following

Theorem 4.3. The Szekeres system (1.1) is completely integrable.

\section{Direct solving of the system (4.1)}

After changing variables, the system (4.1) becomes separable and there arises a chance that it can be also solved directly. Note that two last equations of (4.1) and one of the first integrals $\left(i_{1}\right)$ depend on $\beta$ and $\varepsilon$ only, so now it is possible to investigate the reduced problem

$$
\begin{aligned}
\beta^{\prime} & =\frac{1}{3} \beta^{2}+\frac{1}{2} \varepsilon, \\
\varepsilon^{\prime} & =\varepsilon \beta .
\end{aligned}
$$

The behaviour of the flow on the $\beta O \varepsilon$ plane and at infinity is presented on Fig. 1; the system defined by (5.1) and (5.2) is transformed (see Appendix) to the Poincare disc.

Using the level sets of the first integral $i_{1}=i_{1}(\beta, \varepsilon)$ and the fact that the origin is the only stationary point of the system, we conclude that the system (4.1) admits a family of homoclinic orbits from the origin, contained in the $\beta O \varepsilon$ plane. 
A. Gierzkiewicz and Z. A. Golda / On integrability of the Szekeres system. I

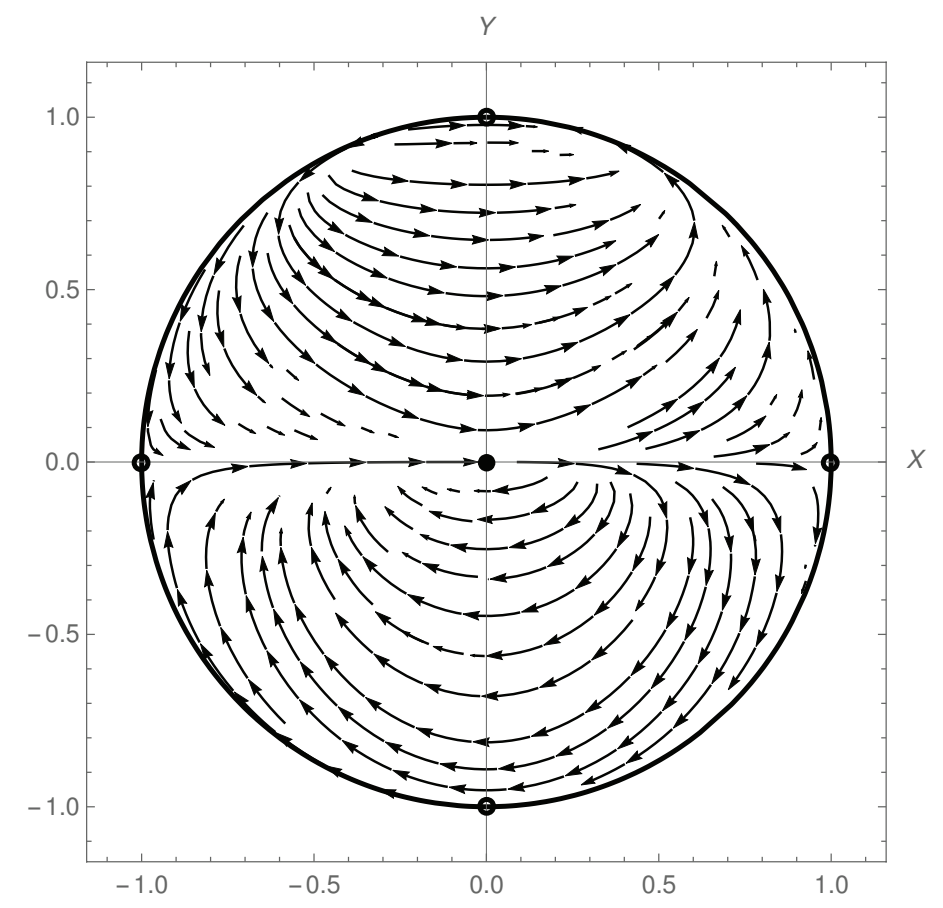

Fig. 1. Dynamics of the system (4.1) on the Poincaré disc of the $\beta O \varepsilon$ plane, transformed to $X O Y$ (see Appendix).

The two-dimensional problem (5.1) and (5.2) can be reduced further to one dimension by setting the first integral $i_{1}=\frac{3 \varepsilon-\beta^{2}}{\varepsilon^{2 / 3}}=$ const. This leads to the substitution

$$
\beta= \pm \sqrt{3 \varepsilon-i_{1} \varepsilon^{2 / 3}}
$$

and then by replacing $\beta$ in (5.2), we obtain an autonomous first-order differential equation

$$
\varepsilon^{\prime}(t)= \pm \varepsilon \sqrt{3 \varepsilon-i_{1} \varepsilon^{2 / 3}}
$$

By integrating we obtain the implicit formula for $\varepsilon=\varepsilon(t)$ :

$$
\begin{array}{ll}
\frac{9 \arctan \left(\frac{\sqrt{-i_{1} \varepsilon^{2 / 3}+3 \varepsilon}}{\sqrt{i_{1}} \varepsilon^{1 / 3}}\right)}{i_{1}^{3 / 2}}+\frac{3 \sqrt{-i_{1} \varepsilon^{2 / 3}+3 \varepsilon}}{i_{1} \varepsilon^{2 / 3}}=t_{0} \pm t & \text { for } i_{1}>0, \quad \text { or } \\
\frac{9 \operatorname{artanh}\left(\frac{\sqrt{-i_{1} \varepsilon^{2 / 3}+3 \varepsilon}}{\sqrt{-i_{1}} \varepsilon^{1 / 3}}\right)}{\left(-i_{1}\right)^{3 / 2}}+\frac{3 \sqrt{-i_{1} \varepsilon^{2 / 3}+3 \varepsilon}}{i_{1} \varepsilon^{2 / 3}}=t_{0} \pm t & \text { for } i_{1}<0,
\end{array}
$$

where $t_{0} \in \mathbb{R}$ is a constant of integration. 
The substitution (5.3) allows us to find directly $\beta$. Subsequently, setting $i_{2}=$ const, one can also substitute

$$
\theta=\frac{-9 \rho-4 \beta^{2}+3 \varepsilon}{6 \beta}+\frac{i_{2} \rho}{\beta \sqrt[3]{\varepsilon}}
$$

to the first equation of (4.1) to obtain a Bernoulli differential equation for $\rho$ :

$$
\rho^{\prime}=\left(\frac{2}{3} \beta-\frac{\varepsilon}{2 \beta}\right) \rho+\left(\frac{3}{2 \beta}-\frac{i_{2}}{\beta \sqrt[3]{\varepsilon}}\right) \rho^{2},
$$

which can be solved in quadratures.

\section{Reparametrized solutions on $\beta O \varepsilon$ plane}

\subsection{Particular solutions}

There are also two particular families of solutions contained in the $\beta O \varepsilon$ plane (i.e. in the set $\{\rho=$ $\theta=0\}$ ) that cannot be put in the form (5.4). These are the solutions contained in the curve $\varepsilon(t)=0$ or $i_{1}=0$.

They are easy to be calculated directly. In order to determine the family of solutions contained in $\varepsilon=0$, we substitute $\varepsilon(t)=0$ to Equation (5.1) and get

$$
\beta^{\prime}(t)=\frac{1}{3} \beta^{2}(t) \quad \Longrightarrow \quad \beta(t)=-\frac{3}{t-t_{0}} \quad \text { or } \quad \beta(t)=0 .
$$

Therefore the first family of solutions consists of $\left(-\frac{3}{t-t_{0}}, 0\right)$, for $t \in\left(-\infty, t_{0}\right)$ or $t \in\left(t_{0}, \infty\right)$ and the stationary solution $(0,0)$ for $t \in \mathbb{R}$.

The other special case is $i_{1}=\frac{3 \varepsilon-\beta^{2}}{\varepsilon^{2 / 3}}=0$, when the solutions' images lie in the curve $\varepsilon=\frac{1}{3} \beta^{2}$ (excluding the point $(0,0)$ ). Substituting this formula to Eq. (5.1), we obtain

$$
\beta^{\prime}(t)=\frac{1}{2} \beta^{2}(t) \quad \Longrightarrow \quad \beta(t)=-\frac{2}{t-t_{0}} \quad \Longrightarrow \quad \varepsilon(t)=\frac{4}{3\left(t-t_{0}\right)^{2}},
$$

so the second family of solutions is $\left(-\frac{2}{t-t_{0}}, \frac{4}{3\left(t-t_{0}\right)^{2}}\right)$, for $t \in\left(-\infty, t_{0}\right)$ or $t \in\left(t_{0}, \infty\right)$.

According to Fig. 2, the curves $\varepsilon=0$ and $\varepsilon=\frac{1}{3} \beta^{2}$ divide the $\beta O \varepsilon$ plane into three areas where the solutions behave differently.

\subsection{Generic solutions}

We are now going to give explicitly all the other solutions on the $\beta O \varepsilon$ plane by reparametrising the independent variable $t$, separately on each of three areas.

Area I: $\left\{3 \varepsilon-\beta^{2}>0\right\}$

Consider a new independent variable $\eta \in(0, \pi)$, related to $t$ through a diffeomorphism

$$
(0, \pi) \ni \eta \longmapsto t(\eta)=\frac{1}{2} \alpha(2 \eta-\sin (2 \eta)) \in(0, \pi \alpha)
$$




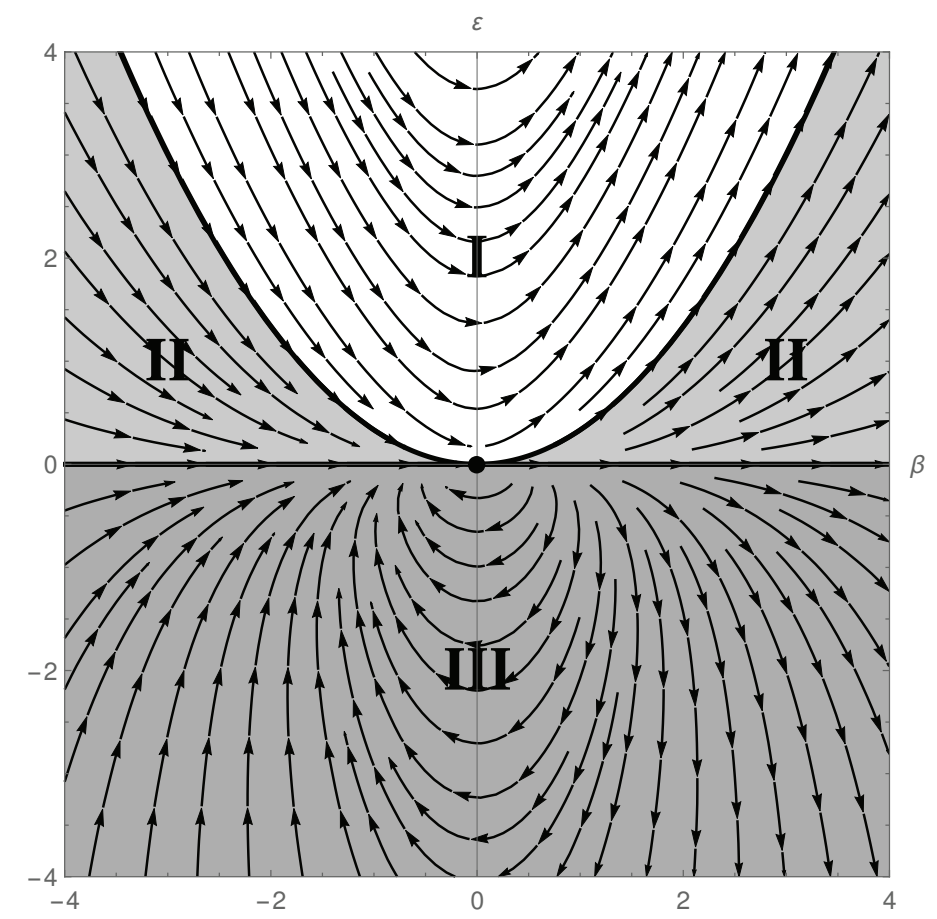

Fig. 2. Dynamics of the system (4.1) on the $\beta O \varepsilon$ plane. Solutions $(\beta(t), \varepsilon(t))$ are divided into three classes: $\varepsilon>\beta^{2} / 3$ (white Area I), $\beta^{2} / 3>\varepsilon>0$ (light gray Area II consisting of two parts), $\varepsilon<0$ (gray Area III).

for some parameter $\alpha>0$. Define also functions

$$
\bar{\beta}(\eta)=-\frac{3 \cot (\eta) \csc ^{2}(\eta)}{\alpha}, \quad \bar{\varepsilon}(\eta)=\frac{3 \csc ^{6}(\eta)}{\alpha^{2}} .
$$

Then there is a family of solutions of (5.1) and (5.2), fulfilling the condition $3 \varepsilon-\beta^{2}>0$, given by the formula

$$
\beta(t)=\bar{\beta}(\eta(t)), \quad \varepsilon(t)=\bar{\varepsilon}(\eta(t))
$$

Note that each solution curve of Area I can be represented in the above form. The solutions are saturated, because $(\bar{\beta}, \bar{\varepsilon}) \rightarrow(-\infty,+\infty)$ with $\eta \rightarrow 0$ and $(\bar{\beta}, \bar{\varepsilon}) \rightarrow(+\infty,+\infty)$ with $\eta \rightarrow \pi$. The curves are symmetric as $\bar{\beta}(\eta)=-\bar{\beta}(\pi-\eta)$ and $\bar{\varepsilon}(\eta)=\bar{\varepsilon}(\pi-\eta)$, with minima $\left(\bar{\beta}\left(\frac{\pi}{2}\right), \bar{\varepsilon}\left(\frac{\pi}{2}\right)\right)=\left(0, \frac{3}{\alpha^{2}}\right)$, and their images cover the Area I as $\alpha \in(0, \infty)$. The first integral is related to the parameter $\alpha$ through $i_{1}=3^{4 / 3} \alpha^{-2 / 3}$.

Area II: $\left\{\beta^{2} / 3>\varepsilon>0\right\}$

Let us define a reparametrisation on this area as

$$
(0, \infty) \ni \eta \longmapsto t(\eta)=\alpha(\sinh (\eta)-\eta) \in \begin{cases}(-\infty, 0) & \text { for } \alpha<0, \\ (0, \infty) & \text { for } \alpha>0 .\end{cases}
$$


Then there is a family of solutions of (5.1) and (5.2), fulfilling the conditions $3 \varepsilon-\beta^{2}<0, \varepsilon>0$, given by $\beta(t)=\bar{\beta}(\eta(t)), \varepsilon(t)=\bar{\varepsilon}(\eta(t))$, where

$$
\bar{\beta}(\eta)=-\frac{3}{\alpha} \frac{\sinh (\eta)}{(\cosh (\eta)-1)^{2}}, \quad \bar{\varepsilon}(\eta)=\frac{3}{4 \alpha^{2}} \operatorname{csch}^{6}\left(\frac{\eta}{2}\right) .
$$

One should observe that for $\alpha<0$, the solutions lie on the left half of the area, because $t \in$ $(-\infty, 0)$, and then $\beta(t)<0$. The solutions on the right part are defined for $\alpha>0$ and their time is positive: $t \in(0,+\infty)$. Let us also mention that the relation between $i_{1}$ and $\alpha$ is $i_{1}=-3^{4 / 3}(2 \alpha)^{-2 / 3}$.

Area III: $\{\varepsilon<0\}$

Define the reparametrisation on the last area as

$$
(-\infty, \infty) \ni \eta \longmapsto t(\eta)=\frac{1}{12} \alpha\left(2 \eta-e^{-\eta}+e^{\eta}\right) \in(-\infty, \infty)
$$

for $\alpha>0$. Then there is a family of solutions of (5.1) and (5.2), fulfilling the condition $\varepsilon<0$, given by $\beta(t)=\bar{\beta}(\eta(t)), \varepsilon(t)=\bar{\varepsilon}(\eta(t))$, where:

$$
\bar{\beta}(\eta)=\frac{36\left(e^{-\eta}\left(e^{-\eta}-1\right)\right)}{\alpha\left(e^{-\eta}+1\right)^{3}}, \quad \bar{\varepsilon}(\eta)=-\frac{1728 e^{-3 \eta}}{\alpha^{2}\left(e^{-\eta}+1\right)^{6}} .
$$

As expected, the solutions are homoclinic to $(0,0)$ :

$$
\lim _{\eta \rightarrow \pm \infty} \bar{\beta}(\eta)=0=\lim _{\eta \rightarrow \pm \infty} \bar{\varepsilon}(\eta)
$$

and symmetric with respect to the $O \varepsilon$ axis, because $\bar{\beta}$ is an odd function and $\bar{\varepsilon}$ is even. The first integral can be expressed as $i_{1}=-9 \alpha^{-2 / 3}$.

\section{Conclusion}

The complete integrability of the Szekeres system (1.1) has been proven by giving the explicit formulas for three independent global first integrals. Moreover, one can find two independent rational integrals. However, the question if the system is completely integrable in the class of rational functions is still open.

It is worth mentioning that we also investigated the more general, six-dimensional Silent Universe system [3] using similar methods. The Darboux polynomials method yields then one first integral only and the Jacobi's last multiplier method cannot be applied. We would also like to indicate that both the Szekeres system and the Silent Universe system have no polynomial integrals. It is a straightforward corollary from the Yoshida theorem $[12,13]$, because one can check that the systems possess balances with the set of negative Kovalevskaya exponents which are not $\mathbb{N}$ independent. This fact can be also proven using the methods similar to those of Llibre and Valls [6]. More on this topic is to be found in our future papers.

\section{Acknowledgments}

The first author would like to thank Professor Jean-Marie Strelcyn for all his devoted time and insightful comments during common discussions.

We also sincerely thank the reviewer for valuable comments that were of great help to us in revising this paper. 


\section{Appendix A. Poincaré compactification}

This well-known method of studying the behaviour of the planar system at infinity by projecting it onto a hemisphere (or further onto the equatorial plane) has been widely discussed in the literature (see [7,9]). Here we shortly present the transformation of the system (5.1) and (5.2) to the Poincaré disc, as shown on Fig. 1.

We start from the system on the plane $\beta O \varepsilon$

$$
\begin{cases}\beta^{\prime}=\frac{1}{3} \beta^{2}+\frac{1}{2} \varepsilon & =: P(\beta, \varepsilon), \\ \varepsilon^{\prime}=\varepsilon \beta & =: Q(\beta, \varepsilon),\end{cases}
$$

and then project it onto the unit sphere, tangent to the plane $\beta O \varepsilon$ at its origin in the point $(0,0,1)$. We use common coordinates $(X, Y, Z)$ on the upper hemisphere $\left\{X^{2}+Y^{2}+Z^{2}=1, Z \geqslant 0\right\}$, so that the relation between them is

$$
\left\{\begin{array} { l } 
{ \beta = \frac { X } { Z } , } \\
{ \varepsilon = \frac { Y } { Z } , }
\end{array} \quad \text { and } \quad \left\{\begin{array}{l}
X=\frac{\beta}{\sqrt{1+\beta^{2}+\varepsilon^{2}}}, \\
Y=\frac{\varepsilon}{\sqrt{1+\beta^{2}+\varepsilon^{2}}}, \\
Z=\frac{1}{\sqrt{1+\beta^{2}+\varepsilon^{2}}} .
\end{array}\right.\right.
$$

We transform the system (A.1) to the system on the upper hemisphere via the formula (s. [9])

$$
\left\{\begin{array}{l}
X^{\prime}=Z\left(\left(1-X^{2}\right) P\left(\frac{X}{Z}, \frac{Y}{Z}\right)-X Y Q\left(\frac{X}{Z}, \frac{Y}{Z}\right)\right), \\
Y^{\prime}=Z\left(\left(1-Y^{2}\right) Q\left(\frac{X}{Z}, \frac{Y}{Z}\right)-X Y P\left(\frac{X}{Z}, \frac{Y}{Z}\right)\right) \\
Z^{\prime}=Z^{2}\left(X\left(-P\left(\frac{X}{Z}, \frac{Y}{Z}\right)\right)-Y Q\left(\frac{X}{Z}, \frac{Y}{Z}\right)\right)
\end{array}\right.
$$

to obtain

$$
\left\{\begin{array}{l}
X^{\prime}=\frac{X^{2}\left(3 Y Z+6 Y^{2}-2\right)+2 X^{4}-3 Y Z}{6 Z} \\
Y^{\prime}=\frac{X Y\left(2 X^{2}+3 Y Z+6 Y^{2}-6\right)}{6 Z} \\
Z^{\prime}=\frac{1}{6} X\left(2 X^{2}+3 Y(2 Y+Z)\right)
\end{array} .\right.
$$

Eventually, we would like to get a two-dimensional system on the Poincare disc $\left\{(X, Y): X^{2}+\right.$ $\left.Y^{2} \leqslant 1\right\}$. It can be done by simply projecting it onto the $X O Y$ plane and setting $Z \mapsto \sqrt{1-X^{2}-Y^{2}}$. We should also rescale it by multiplying by $\sqrt{1-X^{2}-Y^{2}}$ to study the behaviour of the system at infinity. The system on the Poincare disc is of the form (as presented on Fig. 1):

$$
\left\{\begin{array}{l}
X^{\prime}=\frac{1}{6}\left(-2 X^{4}-X^{2}\left(3 Y \sqrt{1-X^{2}-Y^{2}}+6 Y^{2}-2\right)+3 Y \sqrt{1-X^{2}-Y^{2}}\right), \\
Y^{\prime}=-\frac{1}{6} X Y\left(2 X^{2}+3 Y \sqrt{1-X^{2}-Y^{2}}+6 Y^{2}-6\right),
\end{array}\right.
$$

where $(\beta, \varepsilon)$ is transformed to $(X, Y)$.

It can be readily checked that this system involves five stationary points: $(0,0)-$ the only stationary point on $\beta O \varepsilon$ plane - and four points at infinity: $S_{-}=(-1,0), K_{+}=(0,1), S_{+}=(1,0)$, $K_{-}=(0,-1)$. The points $S_{-}, S_{+}$are saddles, whereas $K_{-}, K_{+}$have a more complicated nature, similar to the central degenerate stationary point $(0,0)$, i.e. the Jacobian matrix of the vector field at those points has two conjugated zero eigenvalues. 
A. Gierzkiewicz and Z. A. Golda / On integrability of the Szekeres system. I

\section{References}

[1] K. Bolejko, Structure formation in the quasispherical Szekeres model, Phys. Rev. D 73 (2006) 123508.

[2] K. Bolejko, A. Krasiński, C. Hellaby, M.-N. Célérier, Structures in the Universe by exact methods formation, evolution, interactions, (Cambridge University Press, 2010).

[3] M. Bruni, S. Matarrese and O. Pantano, Dynamics of Silent Universes, Astroph. J. 445 (1995) 958-977.

[4] G. Darboux, Mémoire sur les équations différentielles algébriques du premier ordre et du premier degré (Mélanges), Bull. Sc. Math. 2ème série, 2 (1878) 60-96, 123-144, 151-200.

[5] A. Goriely, Integrability and Nonintegrability of Dynamical Systems, Adv. Series in Nonl. Dyn. 19 (World Scientific, Singapore, 2001).

[6] J. Llibre and C. Valls, Darboux integrability of a generalized Friedmann-Robertson-Walker Hamiltonian system, J. Nonlinear Math. Phys. Vol. 20 No. 3 (2013) 394-406.

[7] S. Lynch, Dynamical Systems with Applications using Mathematica $囚$, (Birkhäuser, Boston, 2007).

[8] Wolfram Research, Inc., Mathematica, ver. 10.0 (Champaign, IL 2015).

[9] L. Perko, Differential Equations and Dynamical Systems, Texts in Applied Mathematics 7 (SpringerVerlag, New York, 2008).

[10] R. A. Sussman and K. Bolejko, A novel approach to the dynamics of Szekeres dust models, Classical and Quantum Gravity 29 (2012) 065018.

[11] P. Szekeres, A Class of Inhomogeneous Cosmological Models, Commun. Math. Phys. 41 (1975) 55-64.

[12] H. Yoshida, Necessary conditions for the existence of algebraic first integrals I: Kowalevski's Exponents, Celest. Mech. 31 (1983) 363-379.

[13] H. Yoshida, Necessary conditions for the existence of algebraic first integrals II: Condition for Algebraic Integrability, Celest. Mech. 31 (1983) 381-399. 